\title{
Metformin-Loaded Nanostructured Plga: A New Strategy for Enhancing Efficacy of Metformin in Breast Cancer Treatment
}

\author{
Mehdi Dadashpour ${ }^{1}$, Monir Yaghoutrang ${ }^{1}$, Mahta Zarghami², Esat \\ Bonabi $^{2}$, Nosratollah Zarghami ${ }^{1,3^{*}}$
}

\begin{abstract}
Objective: Nano-formulation approaches would be more efficient in decreasing drug toxicity, preventing tumor development in relation to free form of drug.

Methods: Therefore, the purpose of this study is to construct poly lactic-coglycolic acid (PLGA) nanoparticles (NPs) loaded with Metformin (MET) to investigate their cytotoxicity as well as their impact on expression levels of apoptosis related genes in MDA-MB-231 breast cancer cells. Field Emission Scanning Electron Microscope (FE-SEM) and Fourier-transform infrared spectroscopy (FTIR) characterized the synthesized NPs. Then, MTT assay was used to evaluate and compare the cytotoxic effect of various concentrations of the chemotherapeutic molecules in pure and nano-formulated forms after $48 \mathrm{~h}$ exposure time. Moreover, the mRNA levels of apoptosis related genes expression were studied by quantitative PCR.

Results: By encapsulating MET into PLGA, the cytotoxic efficiency of the compounds considerably augmented for all concentrations. Furthermore, the results demonstrated that MET-loaded NPs could induce apoptosis in MDAMB-231 breast cancer cell by upregulation of caspase-3, caspase-7 and BAX, along with Bcl-XL, hTERT and Cyclin D1 down regulation.
\end{abstract}

\footnotetext{
${ }^{1}$ Stem Cell Research Center, Tabriz University of Medical Sciences, Tabriz, Iran

${ }^{2}$ Department of Medical Microbiology, Faculty of Medicine, Istanbul Aydin University, Istanbul, Turkey

${ }^{3}$ Department of Clinical Biochemistry and Laboratory Medicine, Faculty of Medicine, Tabriz University of Medical Sciences, Tabriz, Iran

*Corresponding author:

Nosratollah Zarghamizarghami@tbzmed.ac.ir ORCID: 0000-0002-4236-4537

* Received: 13 May 2021 Accepted: 18 June 2021

DOI: 10.17932/EJOH.2020.022/ejoh_v02i1003
} 
Metformin-Loaded Nanostructured Plga: A New Strategy for Enhancing Efficacy of Metformin in Breast Cancer Treatment

Conclusion:Ultimately, thisstudyrevealed that the combinationofMET-PLGANPs withcurrentcancertherapiesholds promise forthepotentialofbreastcancertreatment.

Keywords: Metformin, PLGA, Nanoparticles, MDA-MB-231, Apoptosis

\section{Introduction}

Nowadays, cancer is a major global health issue around the world (1). Breast cancer is considered the most prevalent malignancy, with an estimated 2.3 million new cases (11.7\%) in 2020 and a leading health challenge among females. According to some estimates in recent years, it is responsible for $6.9 \%$ of mortality cases in female breast cancers (2). Despite the significant progression in therapeutic strategies for breast cancer, many patients suffer from relapse and metastasis. The etiology of insufficient therapies can be related to ambiguous insight into molecular mechanisms involved in breast cancer initiation, progression, and metastasis. Multiple genomic and epigenetic events have long been considered hallmarks for the initiation of cancer formation and progression of the disease (3). Current approaches of cancer therapy including radiotherapy and chemotherapy are restricted because of owning various frustrating and unfavorable adverse effects such as unwanted toxicity on the normal cells. Therefore, it is essential that innovative strategies and approaches be developed to effective treatment of human breast cancer (4).

Currently, numerous non-hazardous natural compound have been derived, and their ability in moderating cancer relapse and improvement of treatment have been assessed (5). Some naturals compound like MET is promising products for therapeutic efficiency.

Metformin is a semi-synthetic oral hypoglycemic drug which mostly moderates blood glucose by activating of the vital AMPK/mTOR/p70S6K pathway, inhibiting hepatic glucose output and increasing glucose uptake (6). The anti-cancer ability of MET in various cancer cells including prostate, breast, ovary, cervix cancer, and leukemia incidents both in vivo and in vitro have been studied and recorded (7-9). In spite of the recognized efficacy of MET in treating cancer, the use of this drug in medication is related to some major challenges such as poor biological availability, short half-life, low solubility, low duration of stability in the bloodstream and degradation $(10,11)$. To overcome these limitations, use of nano-formulation system that allows controlled and sustained release of nano-formulated drugs can be a hopeful way to rise the activity and transfer of natural compounds to target cells.

Polymeric particles are a distinctive type of nanomaterials that have found many new applications in delivery systems for in situ controlled release 
of therapeutic applications (12). Nanoparticle drug delivery supplies numerous advantages, due to high encapsulation efficiency, protected drug from degradation, improved biodistribution, delivered, and controlled drug release $(5,13)$

In the current study, we aimed to encapsulate MET into PLGA NPs and investigate the improvement of its anti-cancer effects on MDA-MB-231 breast cancer cells through modulating the expression of apoptosis related gens, as the important molecules involved in tumorigenesis.

\section{Materials and Methods}

\section{Synthesis of NPS}

The improved w/o/w produced MET-encapsulated PLGA NPs with slight modifications (9) . Briefly, a premixed emulsion of PLGA (100 mg) and MET (6 mg) in DCM-Methanol (4:1) was added to the 2\% PVA. The mixture was emulsified by sonication at 70,000 rpm for $1 \mathrm{~min}$ to produce w/o/w emulsion. Then, a rotary evaporator under a low vacuum was used to evaporate the DCM. The NPs formed by this method were gathered via three cycles of centrifugating at $12,500 \mathrm{~g}$ for $15 \mathrm{~min}$.

\section{Nanoparticle characterization}

To characterize the surface charge (zeta potential), hydrodynamic particle size (nm) and polydispersity index (PDI) of designed NPs, dynamic light scattering (DLS) system (Zetasizer Nano ZS; Malvern Instrument) possessing a heliumneon laser beam at $633 \mathrm{~nm}$ wavelength. Furthermore, FE-SEM (Hitachi Ltd., Japan) was exploited to investigate the surface morphological features and Shape of prepared NPs. Also, to further evaluate MET's successful loading on prepared NPs, free MET and NPs, FTIR spectroscopy was utilized to investigate the chemical configuration of the samples in the range of $400-4000 \mathrm{~cm}^{-1}$.

In vitro release study

The MET release pattern from PLGA NPs was obtained through immersing 80 $\mathrm{mg}$ of NPs into $10 \mathrm{~mL}$ of PBS (pH 7.4, $37^{\circ} \mathrm{C}$ ) under shaking at $200 \mathrm{rpm}$. At certain time intervals, $1 \mathrm{~mL}$ of incubation solution was transferred for UV-Vis spectroscopy absorbance measurement at $234 \mathrm{~nm}$ and replaced with $1 \mathrm{~mL}$ of fresh PBS. The cumulative drug release data were plotted as a function of time:

Cumulative amount of release $(\%)=\mathrm{Ct} /(\mathrm{C} \infty \times 100)$

where $\mathrm{Ct}$ is the quantity of MET discharged at time $\mathrm{t}$ and $\mathrm{C} \infty$ refers to total quantity of MET loaded in $100 \mathrm{mg}$ of NPs 
Metformin-Loaded Nanostructured Plga: A New Strategy for Enhancing Efficacy of Metformin in Breast Cancer Treatment

\section{Cell line maintenance}

MDA-MB- 231 breast cancer cell line was gifted from researcher at our university. MDA-MB- 231 cell were grown in RMPI-640 supplemented with 5\% FBS (v/v) and $1 \% \mathrm{pen} / \mathrm{strep}(\mathrm{v} / \mathrm{v})$. The medium was replaced every other to remove floating debris and the flasks were fed with fresh medium

In vitro cytotoxicity

To evaluate the effect of synthesized NPs in increasing the cytotoxicity of MET on MDA-MB- 231, MTT assay was done. First, MDA-MB- 231, at a density of 8,000 cells per well, were seeded into 96-well plates and incubated for $24 \mathrm{~h}$ to become confluent. Then, the cells were treated with various concentrations of MET and MET-NPs and incubated for 24, 48, and $72 \mathrm{~h}$. Then, the medium was aspirated and $50 \mu \mathrm{L}$ MTT solution ( $2 \mathrm{mg} / \mathrm{ml}$, Sigma Aldrich) was added to each well. Next, MTT solution was discarded and replaced with DMSO to dissolve the formazan crystals. The absorbance of solubilized formazan in 96-well plates was measured at a wavelength of $490 \mathrm{~nm}$ using an ELISA plate reader (Dynex MRX).

Real-time PCR assay

Accordingtothemanufacturerinstructions, afterexposure ofA549cellswithdifferent concentrations of the NPs for $48 \mathrm{~h}$, the total RNA was isolated using TRIzol reagent (Invitrogen, USA). Then, a Nanodrop was applied to determine the purity and quantity of total RNA. RevertAid ${ }^{\mathrm{TM}}$ First Strand cDNA synthesis kit (Thermo Fisher Scientific, MA, USA) was used for synthesizing cDNA from $1 \mu \mathrm{g}$ RNA. Next, the quantitative PCR (qPCR) was carried out in a Mic qPCR Cycler (BioMolecular Systems, Australia) by applying SYBR Green Premix PCR Master Mix (Roche, Mannheim, Germany), specific primers, and cDNA. The housekeeping GAPDH gene was applied as the internal control, and the quantification of the samples was analyzed using the $2^{-\Delta \Delta \mathrm{ct}}$ method.

Statistical analysis

All data were presented as mean \pm SD. Statistical analysis was performed using chisquare to determine the statistical significance between the two means evaluated at $\mathrm{p}<0.05$. All experiments were replicated at least three times. Analyses were conducted in GraphPad Prism 8.0 (GraphPad Software, Inc., San Diego, CA).

\section{Results}

\section{Characterization of drug-loaded NPS}

The $\mathrm{O} / \mathrm{W}$ single-emulsion solvent-evaporation process was successfully used for the formulation of the MET-PLGA NPs. The particle size distribution of NPs was assessed by a dynamic light scattering (DLS) method. According to the Table 1, 
the typical particles size, polydispersity, and zeta potential of NPs was $150 \pm 1.17$ $\mathrm{nm}, 0.175$, and zeta potential of $-7.3 \pm 0.27 \mathrm{mV}$, respectively. Drug encapsulated NPs presented larger diameter and a smaller polydispersity than blank NPs. This may include drugs that have amphiphilic copolymers that are completely bound.

Table 1. Mean $( \pm \mathrm{SD})$ particle diameter, Polydispersity $( \pm \mathrm{SD})$ and zeta potential of drug loaded PLGA-PEG NPs.

\begin{tabular}{cccc}
\hline Formulation & $\begin{array}{c}\text { Particle size } \\
(\mathrm{nm})\end{array}$ & $\begin{array}{c}\text { Polydispersity } \\
\text { index }\end{array}$ & $\begin{array}{c}\text { Zeta potential } \\
(\mathrm{mV})\end{array}$ \\
\hline PLGA NPs & $150 \pm 1.17$ & 0.175 & $-7.33 \pm 0.27$ \\
\hline $\begin{array}{c}\text { MET-loaded PLGA/ } \\
\text { PEG NPs }\end{array}$ & $190 \pm 5.56$ & 0.166 & $-6.2 \pm 3.1$ \\
\hline
\end{tabular}

The morphological characterization of MET NPs was studied by SEM. The images of the drug-loaded NPs revealed their regular spherical shape (Figure 1). The surface morphology of NPs was smooth with a regular spherical shape. The size distribution of NPs was narrow with a mean particle diameter $\sim 80 \mathrm{~nm}$. The ranges of drug encapsulation efficiencies (EE) were about $76.8 \%$ with a loading capacity of $12.3 \pm 2.2$, respectively.
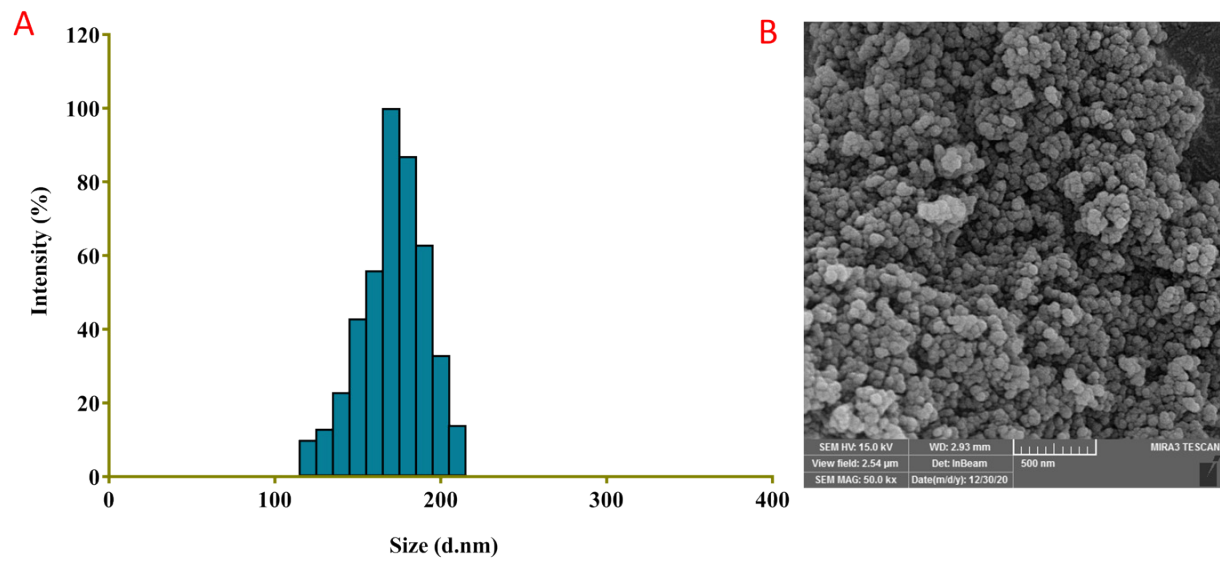

Figure 1. A DLS histogram showing the size distribution of MET NPS. The average size ranged from 130-200 nm. B Field emission scanning electron microscopy (FE-SEM) image of surface morphology of MET-Cur NPS

FTIR was applied to describe the functional groups present in pour and drugs loaded NPs (Fig. 2). The FTIR spectrum of pure MET showed two typical peaks at $3369 \mathrm{~cm}^{-1}$ and $3294 \mathrm{~cm}^{-1}$ relatives to the $\mathrm{N}-\mathrm{H}$ primary stretching 
vibrational mode and a peak at $3155 \mathrm{~cm}^{-1}$ because of the $\mathrm{N}-\mathrm{H}$ secondary stretching, and characteristic peaks at $1626 \mathrm{~cm}-1$ and $1567 \mathrm{~cm}^{-1}$, attributed to C-N stretching. The physical mixture spectrum can be considered as the sum of pure MET and PLGA spectra. The spectrum of MET-loaded NPs shows the typical bands at $3300,3200,1625,1583 \mathrm{~cm}^{-1}$ which are assigned to $\mathrm{N}-\mathrm{H}$ asymmetric stretching, $\mathrm{N}-\mathrm{H}$ symmetric stretching, $\mathrm{C}=\mathrm{N}$ stretching and $\mathrm{N}-\mathrm{H}$ bending groups. The characteristic spectral peaks corresponding to MET functional groups were present in nano-formulations. Also, all the characteristic absorption bands of MET functional groups in the drug loaded polymer, indicating the MET encapsulation into NPs

\section{MET}

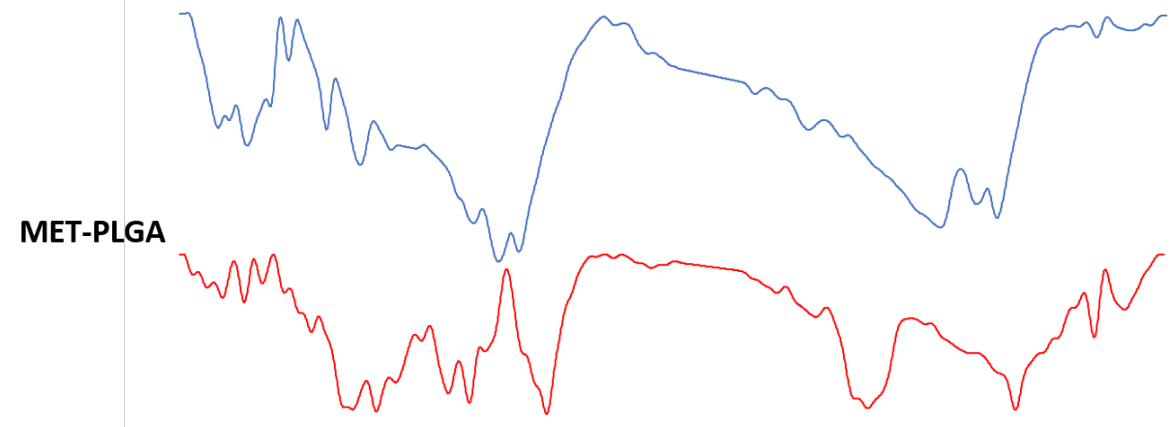

PLGA

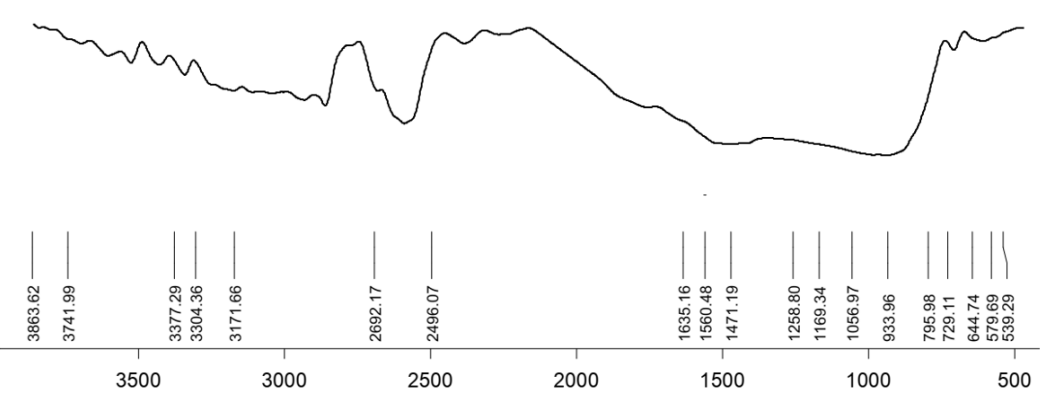

Figure 2. Infrared spectra of MET, PLGA, MET NPS.

Drug release

Cumulative MET release from MET-NPs was assessed for $140 \mathrm{~h}$ at $37{ }^{\circ} \mathrm{C}$. As shown in Fig. 3, MET-NPs exhibited an initial rapid drug discharge pursued by slower constant release rates, which was consistent with the previously reported release kinetics of MET from PLGA NPs. In the first 48 and $72 \mathrm{~h}$, roughly 55.3\% and $72.4 \%$ of MET were released from the PLGA NPs, respectively. 


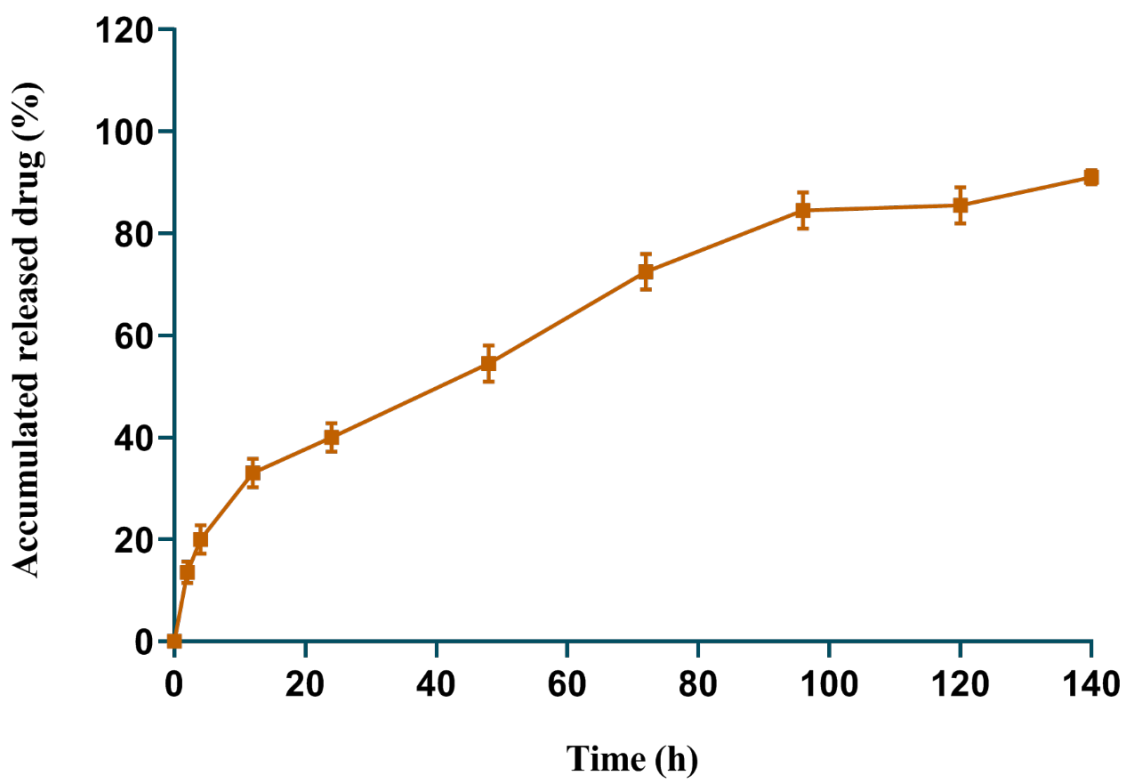

Figure 3. Cumulative release (\%) behavior of MET from NPs in phosphate buffered saline (pH 7.4).

Cell cytotoxicity and synergistic analysis

To evaluate the growth inhibition effect of MET and MET-PLGA NPs, MTT assay was applied using different doses $(0-30 \mathrm{mM})$ of free MET and MET-PLGANPs on MDA-MB-231 breast cancer cells during 24, 48, and 72 h (Fig. 4 and Fig. 5). Cells without treatment were used as control groups.
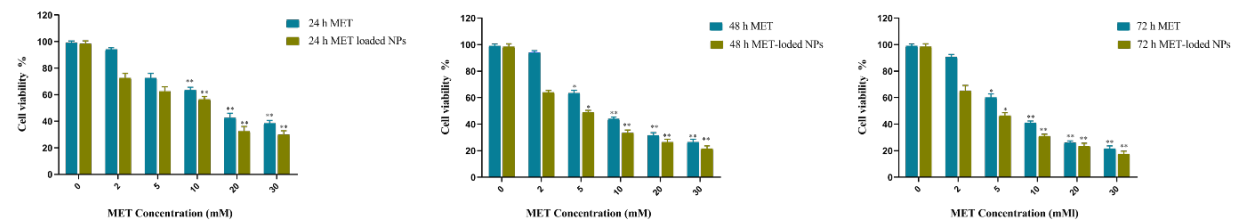

Figure 4. The in vitro cytotoxicity by MTT assay. The viability of $M D A-M B-231$ cells receiving various treatments of free MET and MET-NPs, Cur-NPs. Error bars indicate standard deviations. $\left({ }^{*} p\right.$ value $<0.05, * * p$ value $\left.<0.001\right)$. 


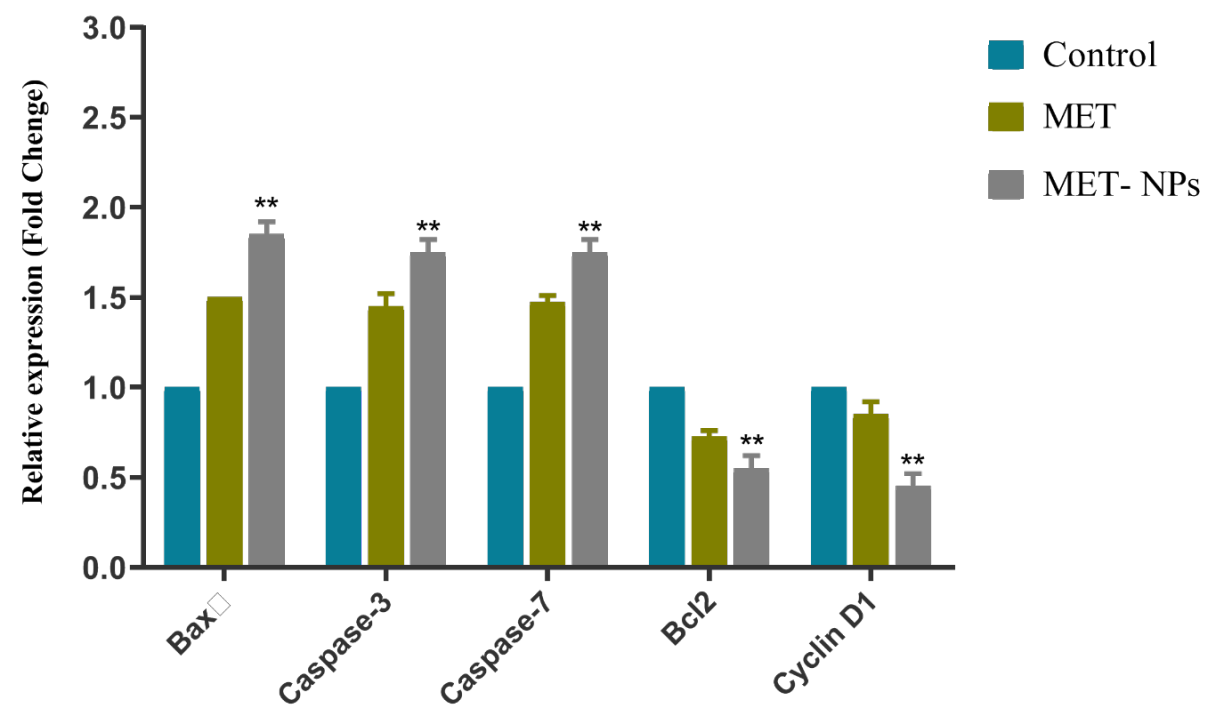

Figure 5. Relative mRNA expression levels of CyclinD1, P53 and apoptosis related genes in MDA$M B-231$ cancer cell line treated with by free and nano-formulated form of MET. ${ }^{*} p<0.05, * * p<0.01$ and $* * * p<0.001$ vs. control was considered significant.

Gene expression findings

The expression of apoptosis related genes including caspase- 3 caspase-7, bcl2, Bax, Cyclin D1 in MDA-MB-231 cancer cell lines after exposure to MET and MET-PLGA NPs for 48 of incubation was determined by real-time PCR. GAPDH region normalized and calculated by the $2^{-\Delta \Delta c t}$ method to assess the alterations in expression levels of apoptosis related genes between the control and treated MDA-MB-231 cancer. Our results showed that MET in free and nanocapsulated forms altered the expression levels of mentioned genes more than free MET and untreated cells. Moreover, our results displayed, caspase-7, caspase-3, and Bax (apoptosis markers) mRNA levels were meaningfully increased in the cells treated with nano-formulated forms of MET compared to control.

\section{Discussion}

As previously described, release of drug from nanoparticles indicates the combined effects of swelling, matrix erosion, degradation processes and in situ cross-linking (14). The sustained and sequential release pattern of chemotherapeutic agents could be valuable for inducing apoptosis and attaining an effective approach in cancer treatment (15). 
As revealed in Fig. 4 in both free and nano forms of MET showed a concentration-dependent growth inhibitory effect on MDA-MB-231 cells. Remarkably, MET-loaded NPs exploited so much higher cytotoxicity relative to the free form of MET against the MDA-MB-231 cells. Improved growth inhibitory activity of drugs in nano-capsulated form might be distributed to the controlled release of drugs from the PLGA NPs and several procedures that included in the high cellular uptake of encapsulated drugs and as a result, leads to the effective discharge of drugs from the PLGA NPs into the cancer cells cytosol.

In one study, the effect of MET- NPs showed a greater inhibitory effect than pure MET by reducing the expression of the hTERT gene in T47D and MDA-MB-231 cell lines in in vitro [16]. Liou and co-colleges showed that MET and SIL in combination form prevent the survival of human cervical cancer cells (17).

Bcl-2 is a member of a substantial family of apoptosis regulatory proteins, which regulate apoptosis by either inducing proapoptotic proteins or inhibiting anti-apoptotic proteins(18). Caspases as a unique family of cysteine proteases are the executive proteins. Caspase- 3 and caspase- 9 are a family of protease enzymes that have a key role in both intrinsic and extrinsic apoptotic pathways (19). The p53 is a tumor suppressor encoded by the gene TP53, and has properties of a transcriptional activator, and it seems to act a role in the regulatory control of normal cellular proliferation. It is also noteworthy that, $\mathrm{p} 53$ contributes directly in the intrinsic path of apoptosis by interacting with the Bcl-2 family that prompt mitochondrial outer membrane permeabilization (20). These observations led to the suggestion that P53/BCL2/BAX apoptosis signaling pathway plays an essential role as a coordinator of apoptosis.

Various studies in consistent with our study have shown that free MET and MET-NPs as well as in combined with other chemotherapeutic molecules can alter the expression levels of apoptosis genes $(16,21)$. In the preliminary study conducted in our group, it was found that MET in combination with SIL exhibits synergistic antiproliferative effects via down-regulating Cyclin D1 and hTERT (16). Moreover, in another study conducted by our group, it was shown that MET loaded into polymeric PLGA-PEG NPs may be a convenient drug delivery system to enhance its anticancer effects for ovarian cancer therapy(9).

In the present study, we proved that delivery of MET in nanoparticles, which are identified as appropriate delivery system for in situ drug delivery and formulation of anticancer drug with low solubility in water leads to strong antiproliferative effect on lung carcinoma cells. 
Metformin-Loaded Nanostructured Plga: A New Strategy for Enhancing Efficacy of Metformin in Breast Cancer Treatment

\section{Conclusions}

In this study, we had successfully fabricated a PLGA-PEG NPs as vehicle to nanocarrier structure to deliver of MET into breast cancer cells for natural based compound chemotherapy. According to the achieved results, MET loaded PLGAPEG NPs where be able of killing MDA-MB-231 breast cancer cells much more than free drug, which is attributing to the sustained and controlled release. Furthermore, MET strongly prevent proliferation of cell and induces apoptosis via reducing of cyclin D1 and bcl-2 mRNA expression levels. In addition, MET increases the RNA transcription level of caspase-3, caspase-7 and Bax as well as p53, resulting in growth inhibition and apoptosis induction. This preliminary study showed that the loading of the chemotherapeutic molecules into PLGA might lead to developing novel and safe drug nano-delivery systems to treat breast cancer effectively.

\section{Acknowledgements}

The authors would like to thank the Stem Cell Research Center for the use of their laboratory facilities.

\section{Declaration of Competing Interest}

The authors declare that they have no competing interests.

\section{References}

1. Clinton SK, Giovannucci EL, Hursting SD. The World Cancer Research Fund/American Institute for Cancer Research Third Expert Report on Diet, Nutrition, Physical Activity, and Cancer: Impact and Future Directions. The Journal of Nutrition 2020;150:663-671.

2. Sung H., et al. Global cancer statistics 2020: GLOBOCAN estimates of incidence and mortality worldwide for 36 cancers in 185 countries. CA: A Cancer Journal for Clinicians. $\mathrm{n} / \mathrm{a}(\mathrm{n} / \mathrm{a})$.

3. Riggio AI, Varley KE, Welm AL, The lingering mysteries of metastatic recurrence in breast cancer. British Journal of Cancer 2021;124:13- 26.

4. Lotfi-Attari J, et al., Co-delivery of curcumin and chrysin by polymeric nanoparticles inhibit synergistically growth and hTERT gene expression in human colorectal cancer cells. Nutrition and cancer 2017;69:1290- 1299.

5. Farajzadeh R, et al. Nano-encapsulated metformin-curcumin in PLGA/PEG inhibits synergistically growth and hTERT gene expression in human breast cancer cells. Artificial cells, nanomedicine, and biotechnology 2018;46:917- 
Mehdi Dadashpour, Monir Yaghoutrang, Mahta Zarghami, Esat Bonabi, Nosratollah Zarghami

925.

6. Jafari-Gharabaghlou D, et al. Combination of metformin and phenformin synergistically inhibits proliferation and hTERT expression in human breast cancer cells. Iranian Journal of Basic Medical Sciences 2018;21:1167.

7. Saraei $\mathrm{P}$, et al. The beneficial effects of metformin on cancer prevention and therapy: a comprehensive review of recent advances. Cancer management and research 2019;11:3295.

8. Chatran M, et al. Synergistic anti-proliferative effects of metformin and silibinin combination on T47D breast cancer cells via hTERT and cyclin D1 inhibition. Drug Res 2018;68:710-716.

9. Faramarzi L, et al. Enhanced anti-proliferative and pro-apoptotic effects of metformin encapsulated PLGA-PEG nanoparticles on SKOV3 human ovarian carcinoma cells. Artificial Cells, Nanomedicine, and Biotechnology 2019; 47:737-746.

10. Bhandare N, Narayana A. Applications of nanotechnology in cancer: a literature review of imaging and treatment. J Nucl Med Radiat Ther 2014;5:19.

11.Hrkach JS, et al. Nanotechnology for biomaterials engineering: structural characterization of amphiphilic polymeric nanoparticles by $1 \mathrm{H}$ NMR spectroscopy. Biomaterials 1997;18:27-30.

12. Akbarzadeh Khiavi M, Safary A, Somi MH. Recent advances in targeted therapy of colorectal cancer: impacts of monoclonal antibodies nanoconjugates. BioImpacts: BI. 2019;9:123-127.

13. Dadashpour M, et al. Biomimetic synthesis of silver nanoparticles using Matricaria chamomilla extract and their potential anticancer activity against human lung cancer cells. Mater Sci Eng C Mater Biol Appl 2018;92:902-912. doi: 10.1016/j.msec.2018.07.053.

14. Wu H, et al. Chitosan-polycaprolactone copolymer microspheres for transforming growth factor- $\beta 1$ delivery. Colloids Surf B Biointerfaces 2011; 82: 602-8. doi: 10.1016/j.colsurfb.2010.10.024.

15. Senapati S, et al. Controlled drug delivery vehicles for cancer treatment and their performance. Signal transduction and targeted therapy, 2018;3:1-19.

16. Amirsaadat $\mathrm{S}$, et al. metformin and Silibinin co-loaded PLGA-PEG nanoparticles for effective combination therapy against human breast cancer cells. Journal of Drug Delivery Science and Technology 2021;61:102107.

17. Liou WS, et al. Herbal product silibinin-induced programmed cell death is 
enhanced by metformin in cervical cancer cells at the dose without influence on nonmalignant cells. Journal of Applied Biomedicine, 2015;13:113- 121.

18. Baharara J. et al. Silver nanoparticles biosynthesized using Achillea biebersteinii flower extract: apoptosis induction in MCF-7 cells via caspase activation and regulation of $\mathrm{Bax}$ and $\mathrm{Bcl}-2$ gene expression. Molecules 2015;20: 2693-2706.

19. El-Aal AAA, et al. Post-immunization immunohistochemical expression of Caspase 3 and p53 apoptotic markers in experimental hydatidosis. Revista Brasileira de Parasitologia Veterinária 2016;25:333-340.

20. Aubrey BJ, et al. How does p53 induce apoptosis and how does this relate to p53-mediated tumour suppression? Cell Death \& Differentiation 2018;25:104113.

21. Samadzadeh $\mathrm{S}$, et al. An implantable smart hyperthermia nanofiber with switchable, controlled and sustained drug release: possible application in prevention of cancer local recurrence. Materials Science and Engineering:C 2021;118:111384.48 